dent on achieving a good antipneumococcal antibody response and a serum antibody nitrogen concentration greater than $300 \mathrm{ng} / \mathrm{ml}$ is believed to offer protection for two to five years. ${ }^{3}$ If possible vaccination should occur two weeks before splenectomy as higher antibody titres are produced. When a splenectomy is performed as an emergency such as after abdominal trauma, however, vaccination should still be performed because the antibody titres achieved are considered to be protective in most cases.

Although vaccination is generally thought to be effective, overwhelming pneumococcal sepsis in previously vaccinated patients does occur. ${ }^{6}$ This may be due to several factors: (a) not all pneumococcal subtypes are covered in the vaccine; (b) the subtypes do not produce equal titres of antipneumococcal antibodies; and (c) the antibody titres, which may originally have been adequate, may decrease with time exposing the patient to pneumococcal infection.

Because of the latter reason revaccination of high risk adult patients has been performed with few side effects. ${ }^{4}$ In children, however, revaccination is not currently recommended within six years of the primary vaccination. ${ }^{4}$ Primary vaccination in children under 2 years of age is currently not recommended because of poor antibody production.

The Pneumovax II vaccine fails to cover all the pneumococcal subtypes. The loss of protection with time after vaccination because of the falling antipneumococcal antibody titres, repeated measurements of which in high risk patients would be logistically difficult to organise, means there is a need for a second concurrent prophylactic measure.
Prophylactic daily penicillin, which would theoretically cover all the pneumococcal subtypes, has been shown to be effective in reducing the incidence of pneumococcal sepsis in postsplenectomy patients. Its indefinite prophylactic use has been recommended in one study. ${ }^{2}$ Poor compliance, allergic reactions, and the risk of producing penicillin resistant pneumococcal strains are the main problems cited with this approach.

Because of the previous reports on overwhelming postsplenectomy infection, ${ }^{13}$ together with our own experience, we strongly recommend for all children who undergo splenectomy:

(1) Vaccination with Pneumovax II and revaccination at intervals of six years.

(2) Continuation of penicillin indefinitely or at least until the child has left an environment where there is an increased epidemiological risk of pneumococcal infection, such as school, university, or the armed forces.

(3) Continual parental awareness and vigilance.

1 Dickerman JD. Splenectomy and sepsis: a warning. Pediatrics 1979;63:938-41.

2 Zarrabi MH, Rosner F. Rarity of failure of penicillin prophylaxis to prevent postsplenectomy sepsis. Arch Intern Med 1986;146:1207-8.

3 Bolan G, Broome CV, Facklam RR, Pilaytis BD, Fraser DW, Schlech WF. Pneumococcal vaccine efficacy in selected populations in the United States. Ann Intern Med 1986;104: 1-6.

4 Anonymous. When to use the new pneumococcal vaccine. Drug Ther Bull 1990;28:31-2.

5 Shann F. Modern vaccines: pneumococcus and influenza. Lancet 1990;335:898-901.

6 Evans DIK. Fatal postsplenectomy sepsis despite prophylaxis with penicillin and pneumococcal vaccine. Lancet 1984;i: 1124.
Royal Hospital for Sick Children, Yorkhill, Glasgow G3 8SJ $P$ Galea K M Goel

Correspondence to: Dr Galea.

Accepted 13 June 1990

(Arch Dis Child 1990;65:1269-70).

\title{
Visceral leishmaniasis in a Scottish child
}

\author{
P Galea, K M Goel
}

\begin{abstract}
A Scottish girl acquired visceral leishmaniasis (kala-azar) while on holiday in Majorca. She presented with the infection, six months later, in Scotland. Because of inexperience with the disease and a degree of scepticism unnecessary investigations were carried out resulting in a delay in treatment.
\end{abstract}

Visceral leishmaniasis (kala-azar) is found in an area extending from the Straits of Gibraltar, across the Mediterranean through Asia to China. The vector is a sandfly, which transmits the infection from the natural reservoir usually an infected dog, to human beings during feeding. The sandfly does not survive in the colder climates north of the Mediterranean and leishmaniasis is virtually unknown in these countries. ${ }^{1}$ Because of its long incubation period (six weeks to three years), however, it is possible for kala-azar acquired in an endemic area to present in a country where the disease is not endemic.
We describe the case of a Scottish girl who acquired kala-azar while on holiday in a Mediterranean country and presented with the infection in Scotland.

\section{Case report}

A 9 month old girl was admitted to the Royal Hospital for Sick Children, Glasgow, with a 10 day history of lethargy, pallor, fever, and poor feeding. On examination she was found to have a mass in the left hypochondrium. She was febrile and miserable. Her haemoglobin concentration was $65 \mathrm{~g} / 1$, white cell count $6.3 \times 10^{9} / 1$, and platelets $41 \times 10^{9} / 1$. Initially she was thought to be suffering from a malignant condition and was investigated accordingly. Bone marrow examination, blood culture, chest radiography, skeletal survey, and urine catecholamines were all normal. Abdominal ultrasound examination showed the mass to be a massively enlarged spleen.

She was given a blood transfusion and antibio- 


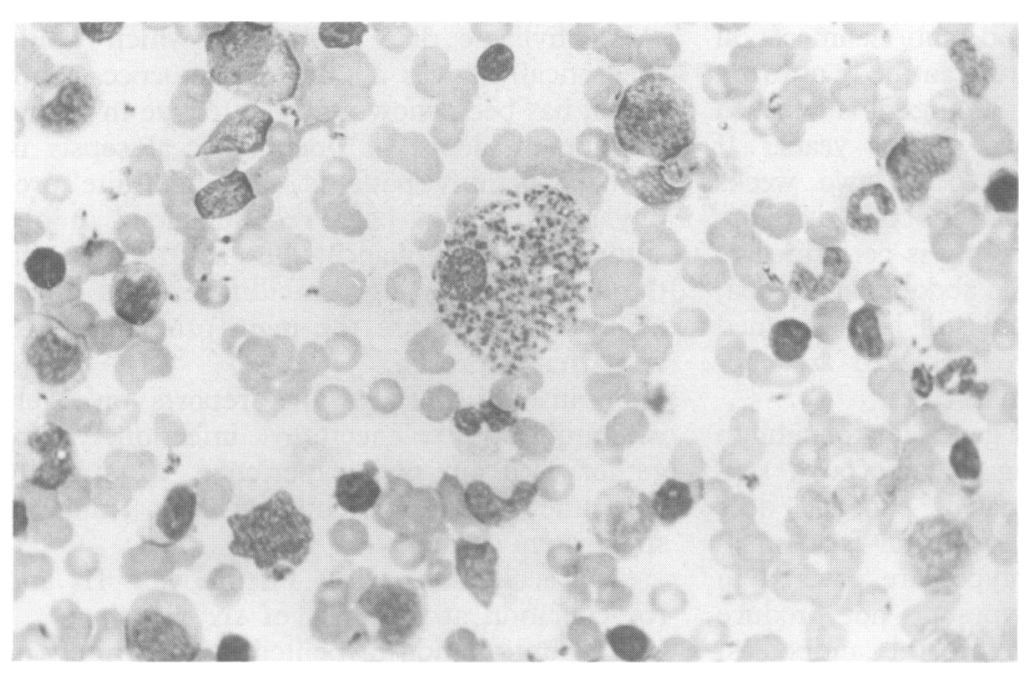

Bone marrow showing presence of Leishman-Donovan bodies

tics after which her general condition improved, although she continued to spike a fever two to three times a day. By the third day after admission, the results of the above investigations were all negative and the possibility of visceral leishmaniasis was raised. The child had been on holiday in Majorca six months earlier. The bone marrow was re-examined and again thought to be normal. Leishmania antibody titres were negative and as the condition was considered to be highly unlikely in a Scottish girl the diagnosis was discarded.

The girl remained febrile with increasing splenomegaly and hepatomegaly. Thrombocytopenia persisted and she also became neutropenic and was started on a neutropenic regimen.

Her fever did not respond to treatment with various antibiotics and repeated blood cultures were negative. Viral antibody titres to cytomegalovirus and parvovirus were negative as was her HIV antibody titre.

Three weeks after admission a bone marrow trephine was performed. A sample was sent to the
London School of Hygiene and Tropical Medicine where the presence of Leishman-Donovan bodies was noted (Dr PL Chiodinie, consultant parasitologist). Re-examination of the original bone marrow also showed the presence of Leishman-Donovan bodies (figure).

The girl was treated with sodium stibogluconate $20 \mathrm{mg} / \mathrm{kg} /$ day for 10 days followed by 10 $\mathrm{mg} / \mathrm{kg} /$ day for another 10 days. Her temperature settled within two days of starting treatment. Her platelet count returned to normal within seven days and by the 10th day of treatment her white cell count reached normal values.

She made an uneventful recovery and one year after completing treatment she remains perfectly well. Leishmania serology first became positive five weeks after presentation.

\section{Discussion}

We believe this to be the first case of kala-azar to be diagnosed in a child in Scotland. Because of inexperience with the disease and a degree of scepticism regarding the diagnosis, treatment of the condition was delayed by three weeks during which time the girl was subjected to a number of unnecessary investigations. In these days of global travel, with so many British tourists spending their holidays in the Mediterranean, it is likely that more cases of leishmaniasis will be seen in Britain because of the long incubation period.

The condition should be included in the differential diagnosis of any child or young adult, presenting with massive splenomegaly, severe anaemia, and thrombocytopenia who has been in an endemic area up to three years previously. Negative leishmania serology may not exclude the diagnosis during the initial stages of the disease as it may take a few weeks before becoming positive.

1 Feigin R, Cherry JD. Textbook of paediatric infectious diseases. Philadelphia: W B Saunders, 1987:2047-8.

\title{
Crohn's disease of the lung
}

\author{
J W L Puntis, M J Tarlow, F Raafat, I W Booth
}

Institute of Child Health,
University of
Birmingham
J W L Puntis
I J Tarlow
The Children's Hospital,
Birmingham
F Raafat
Correspondence to:
Dr J W L Puntis,
Department of Paediatrics
and Child Health,
University of Leeds,
Clarendon Wing,
The General Infirmary,
Belmont Grove,
Leeds LS2 9NS.
Accepted 1 July 1990

(Arch Dis Child 1990;65:1270-1).

\section{Abstract}

Two years after developing colonic Crohn's disease, a 17 year old boy presented with focal pulmonary consolidation. A lung biopsy specimen showed areas of non-caseating epithelioid granuloma. Although some respiratory abnormalities appear to be associated with inflammatory bowel disease, granulomatous disease affecting the lung has not previously been reported in a child.

Crohn's disease appears to be a multisystem disorder, ${ }^{1}$ but granulomatous pulmonary involvement has not been previously reported. We present our experience with a teenage boy.

\section{Case report}

A 15 year old boy was referred to hospital with a short history of diarrhoea, weight loss, and abdominal pain. Other features suggestive of inflammatory bowel disease included arthralgia, perianal inflammation, and erythema multiforme. At colonoscopy, a moderately severe pancolitis was found. Mucosal biopsy specimens were typical of Crohn's disease, showing non-caseating granuloma with epithelioid and histiocytic cells admixed with multinucleated giant cells (fig 1). The patient remained symptom free on maintenance treatment with mesalazine $\mathbf{4 0 0} \mathrm{mg}$ three times a day, but two years later, developed left sided pleuritic chest pain, intermittent fever, and lassitude. A chest radiograph showed 